\title{
Recognition and treatment of peri-implant mucositis: Do we have the right perception? A structured review
}

\author{
Laura Lo Bianco ${ }^{1, A, C-E}$, Marco Montevecchi ${ }^{1, D, E}$, Michele Ostanello $0^{1, B, D}$, Vittorio Checchi ${ }^{2, D-F}$ \\ 1 Department of Biomedical and Neuromotor Sciences, Dental School, University of Bologna, Italy \\ ${ }^{2}$ Unit of Dentistry and Oral \& Maxillofacial Surgery, Surgical, Medical and Dental Department of Morphological Sciences related to Transplant, Oncology and Regenerative Medicine, \\ University of Modena and Reggio Emilia, Modena, Italy
}

A - research concept and design; $\mathrm{B}$ - collection and/or assembly of data; $\mathrm{C}$ - data analysis and interpretation;

$\mathrm{D}$ - writing the article; $\mathrm{E}$ - critical revision of the article; $\mathrm{F}$ - final approval of the article

Address for correspondence

Laura Lo Bianco

E-mail: laura_10_bianco@hotmail.com

Funding sources

None declared

Conflict of interest

None declared

Received on January 16, 2021

Reviewed on April 28, 2021

Accepted on May 4, 2021

Published online on December 28, 2021

\begin{abstract}
Peri-implant mucositis is a common inflammatory lesion of the soft tissues surrounding endosseous implants, with no loss of the supporting bone. Its prevention or early diagnosis are vital for dental implant success.

The aim of this review was to investigate knowledge strengths and gaps in clinicians' perceptions of periimplant mucositis prevalence and evidence for successful treatment.

A literature search for articles published until 2020, reporting on the prevalence of peri-implant mucositis and its treatment was performed in standard online databases. The inclusion criteria were as follows: studies in English; studies with an available abstract; studies on humans with at least 1 dental implant; and studies reporting on the prevalence and/or treatment of peri-implant mucositis. Sixty-five studies fulfilled the inclusion criteria. The included papers were analyzed to identify data on the prevalence and treatment of peri-implant mucositis. The prevalence statistics for peri-implant mucositis had wide ranges in both the patient-based (PB) analysis and the implant-based (IB) analysis; the possible reasons for these wide ranges are discussed. Treatment methods for peri-implant mucositis were analyzed individually and compared to the management of gingivitis.

It was determined that the currently available information on the prevalence rates and the standardized therapeutic protocols for peri-implant mucositis are insufficient. Since the mean gingivitis and peri-implant mucositis prevalence rates in the $\mathrm{PB}$ analysis were similar, it is possible that peri-implant mucositis is underestimated due to variables related to implant rehabilitation itself.
\end{abstract}

Keywords: inflammation, dental implant, literature review, oral mucositis, peri-implant healing

Cite as

Lo Bianco L, Montevecchi M, Ostanello M, Checchi V. Recogni-

tion and treatment of peri-implant mucositis: Do we have

the right perception? A structured review. Dent Med Probl.

2021;58(4):545-554. doi:10.17219/dmp/136359

DOI

$10.17219 / \mathrm{dmp} / 136359$

Copyright

○) 2021 by Wroclaw Medical University

This is an article distributed under the terms of the

Creative Commons Attribution 3.0 Unported License (CC BY 3.0)

(https://creativecommons.org/licenses/by/3.0/). 


\section{Introduction}

Dental implants are widely used for oral rehabilitation. They are biocompatible prosthetic devices implanted in living bone and, for this reason, the peri-implant tissue conditions can change over time. ${ }^{1,2}$ Healthy peri-implant tissues are characterized by the absence of erythema, bleeding on probing (BoP), swelling, and suppuration. ${ }^{3}$

Once osteointegration has been achieved, allowing for the healing time after implant insertion, implant complications can occur due to mechanical problems, inflammation and/or the loss of the surrounding tissues (the oral mucosa and the supporting bone). These could lead to relevant discomfort for the patient as well as implant failure over time. ${ }^{4}$ After osteointegration has occurred, implants may become contaminated and peri-implant tissues could become inflamed, causing peri-implant mucositis and/or peri-implantitis. ${ }^{3}$

In an animal study on beagle dogs, Berglundh et al. compared the anatomy and histology of peri-implant and periodontal tissues in block biopsies..$^{5} \mathrm{~A}$ histological examination showed that both presented well-keratinized areas (the oral epithelium and the outward portion of the peri-implant mucosa), but periodontal tissues presented only a few cells of thick epithelium in contact with the implant abutment. Also, peri-implant tissue fibers displayed a parallel course originating from the crestal bone, while periodontal tissue fibers were perpendicular to the dental root, going from the root cementum to the alveolar bone. ${ }^{5}$

Likewise, blood supply differed from an anatomical point of view - the peri-implant bone vasculature consisted only in the periosteum source, while gingival supply was guaranteed by a double source composed of supraperiosteal and periodontal ligament vessels. ${ }^{6}$

Being aware of histological differences between periimplant tissues and the periodontium is fundamental to better understand the peri-implant tissue biology. Clinicians, implant-rehabilitated patients and the dental industry have mainly based their maintenance approaches on the techniques and tools derived from the pre-implant era.

Peri-implant mucositis is an inflammatory lesion of the soft tissues surrounding an endosseous implant, with no loss of the supporting bone or the continuing marginal bone. ${ }^{7}$ Conversely, peri-implantitis is described as a pathological condition occurring in tissues around dental implants that is characterized by inflammation in the periimplant connective tissue and a progressive loss of the supporting bone. ${ }^{8}$

The etiology of peri-implant mucositis has been described as the accumulation of bacterial biofilm around the implant, which may cause signs and symptoms of inflammation, such as local swelling, redness, pain, and BoP. ${ }^{7}$ The diagnosis of peri-implant mucositis vs. peri-implantitis is made by the evidence of pathological bone loss. ${ }^{9}$ While peri-implant mucositis exhibits signs of inflammation with no bone loss besides the remodeling process of the alveolar bone during the first year after implantation, peri-implantitis shows signs of inflammation associated with a further loss of the crestal bone. ${ }^{3,7}$ In recent years, there has been a general consensus that following the first year of implant functioning, bone loss around dental implants $\geq 2 \mathrm{~mm}$ represents periimplantitis. ${ }^{10}$

Data indicates that patients diagnosed with periimplant mucositis may develop peri-implantitis, especially in the absence of regular maintenance care, but the processes and reasons for this pathological progress remain unknown. ${ }^{11}$ Factors associated with peri-implant mucositis include biofilm accumulation, smoking and radiation therapy. ${ }^{7}$ Regular supportive peri-implant care with biofilm removal is an important preventive strategy against the conversion of a healthy tissue to peri-implant mucositis, and also against the progression of peri-implant mucositis into peri-implantitis. ${ }^{7,11}$

There is evidence that peri-implant mucositis can be successfully treated. The resolution of the clinical signs of inflammation may take more than 3 weeks following the restoration of plaque/biofilm control. ${ }^{11}$ The management of peri-implant inflammation should be addressed in terms of infection control, decontamination of the implant surface and regeneration of the alveolar bone when needed. ${ }^{12}$

The early diagnosis and prevention of peri-implant infections are essential for the long-term dental implant success. In order to perform a thorough evaluation of the peri-implant conditions, peri-implant probing and relative radiographs are always required. ${ }^{12,13}$

The purpose of this review was to highlight possible clinicians' perception problems related to peri-implant mucositis, to investigate the prevalence of peri-implant mucositis reported in the literature and to analyze the evidence-based data regarding its treatment.

\section{Material and methods}

\section{Focus question}

The focus question for the literature search was: "What is the clinician's perception regarding the prevalence levels and treatment strategy efficacy/evidence for periimplant mucositis?"

It was structured according to the PICO format ${ }^{14}$ :

- Population: patients rehabilitated with dental implants;

- Intervention: implant prosthesis, peri-implant tissue, and peri-implant mucositis prevalence and treatment;

- Comparison: diagnostic criteria and peri-implant mucositis treatment;

- Outcome: finding consistency between prevalence and perception, and differences between various kinds of treatment. 


\section{Search strategy}

The PubMed/MEDLINE, Embase, Scopus, Web of Science, and Cochrane databases were searched to identify published articles reflecting the inclusion criteria: studies in English; studies with an available abstract; studies involving humans with at least 1 dental implant; and studies reporting data on the prevalence and/or treatment of peri-implant mucositis. The search strategy was divided into 2 parts: a pre-search to avoid discrepancies between findings due to the device used (a personal computer or a mobile device); and a focus question search.

The pre-search was used to determine the device and keywords that provided the greatest number of results in order to establish the focus question search. The presearch concerned peri-implant mucositis studies published up to 2020. The terms used for the identification of keywords were: 'peri implant' OR 'peri-implant' OR 'peri-implant mucositis' AND 'mucositis'.

The focus question search was carried out on a personal computer to analyze the abovementioned databases, using the 2 keywords that yielded the greatest number of results in the pre-search. The focus question search concerned peri-implant mucositis studies published up to 2020. The terms used for the identification of keywords were: 'peri implant mucositis' OR 'peri-implant mucositis' AND 'prevalence' OR 'treatment'.

The focus question search yielded 99 articles for "peri implant mucositis prevalence", 99 for "peri-implant mucositis prevalence", 300 for "peri implant mucositis treatment", and 271 for "peri-implant mucositis treatment".

\section{Screening and selection}

The inclusion criteria were as follows: studies in English; studies with an available abstract; studies involving humans with at least 1 dental implant; and studies reporting on the prevalence and/or treatment of peri-implant mucositis.

The exclusion criteria were as follows: studies in a language other than English; studies without an available abstract; non-clinical studies; studies without dental implants; and studies reporting on neither the prevalence nor the treatment of peri-implant mucositis.

Once the studies were selected according to the abovementioned initial screening, only those fitting the following categories were included: randomized clinical trials (RCTs); controlled clinical trials (CCTs); cohort studies; cross-sectional studies; and case-control studies.

The studies were first screened by titles and abstracts, and examined by 2 reviewers. The full text of the selected articles was retrieved and the study results were analyzed. Review articles and systematic reviews were also studied in order to find other articles that did not emerge during database inquiries.

Full-text studies admitted for final analysis were divided into 2 groups: the prevalence group; and the treatment group.

\section{Results}

Sixty-five studies fulfilled all the inclusion criteria: 25 RCTs; 3 CCTs; 15 cohort studies; 20 cross-sectional studies; and 2 case-control studies. All these studies were divided into 2 main groups according to the 'prevalence' $(n=34)$ or 'treatment' $(n=31)$ Medical Subject Headings $(\mathrm{MeSH})$. The results according to the type of study are shown in Table 1 for the prevalence group and in Table 2 for the treatment group.

In the prevalence group, cohort and cross-sectional studies constituted the majority of the research devoted to peri-implant mucositis (Table 1). In cohort studies, the peri-implant mucositis prevalence rates ranged between $7.14 \%$ and $68.00 \%$ in the patient-based (PB) analysis (referring to the number of patients included in the analysis), and between $5.06 \%$ and $38.00 \%$ in the implant-based (IB) analysis (referring to the number of implants included in the analysis). In cross-sectional studies, the peri-implant mucositis prevalence ranges varied from $20.80 \%$ to $80.90 \%$ in the PB analysis, and from $21.00 \%$ to $90.00 \%$ in the IB analysis (Table 3 ).

In the treatment group, there were RCTs, CCTs, cohort studies, and 1 case-control study (Table 2). The search found 1 RCT on the use of sodium hypochlorite gel, $1 \mathrm{RCT}$ about the modification of the prosthesis, $1 \mathrm{RCT}$ on the use of a drying agent associated with manual debridement, 2 RCTs in which chlorhexidine gel was used, 1 RCT

Table 1. Prevalence group results according to the type of study

\begin{tabular}{|l|c|}
\multicolumn{1}{l}{ Type of study } & Number of articles \\
\hline RCTs & 0 \\
CCTs & 0 \\
Cohort studies & 13 \\
Cross-sectional studies & 20 \\
Case-control studies & 1 \\
\hline
\end{tabular}

RCT - randomized clinical trial; CCT - controlled clinical trial,

Table 2. Treatment group results according to the type of study

\begin{tabular}{|c|c|}
\hline Type of study & Number of articles \\
\hline RCTs & 25 \\
\hline CCTs & 3 \\
\hline Cohort studies & 2 \\
\hline Cross-sectional studies & 0 \\
\hline Case-control studies & 1 \\
\hline
\end{tabular}

Table 3. Peri-implant mucositis prevalence ranges according to the type of study

\begin{tabular}{|l|c|c|}
\multirow{2}{*}{\multicolumn{1}{c}{ Type of study }} & \multicolumn{2}{|c}{$\begin{array}{c}\text { Prevalence range } \\
\text { [\%] }\end{array}$} \\
\cline { 2 - 3 } & PB analysis & IB analysis \\
\hline Cohort studies & $7.14-68.00$ & $5.06-38.00$ \\
Cross-sectional studies & $20.80-80.90$ & $21.00-90.00$ \\
\hline
\end{tabular}

PB - patient-based; IB - implant-based. 
Table 4. Treatment proposed with regard to the type of study

\begin{tabular}{|c|c|}
\hline Type of study & Treatment tested \\
\hline $\begin{array}{l}\text { RCTs, CCTs and } \\
\text { a case-control study }\end{array}$ & $\begin{array}{l}\text { sodium hypochlorite gel } \\
\text { modifying the prosthesis } \\
\text { desiccant agent } \\
\text { chlorhexidine gluconate } \\
\text { cetylpyridinium } \\
\text { triclosan } \\
\text { chitosan brushes } \\
\text { probiotics } \\
\text { diode laser } \\
\text { photodynamic therapy } \\
\text { air polishing } \\
\text { enamel matrix derivative } \\
\text { ozone } \\
\text { hydrogen peroxide } \\
\text { systemic antibiotics } \\
\text { azithromycin } \\
\text { mechanical curettage }\end{array}$ \\
\hline Cohort studies & non-surgical therapy \\
\hline
\end{tabular}

in which a mouth rinse with $0.03 \%$ chlorhexidine and $0.05 \%$ cetylpyridinium was assessed, 1 RCT that investigated $0.12 \%$ chlorhexidine gluconate, 3 RCTs in which toothpastes containing triclosan were assessed, 1 RCT in which chitosan brushes were used, 5 RCTs about probiotics (in one of the studies, photodynamic therapy was added to probiotic administration), 2 RCTs about photodynamic therapy, 3 RCTs about air polishing, 1 RCT in which an enamel matrix derivative was used, 1 RCT on the use of ozone and/or hydrogen peroxide, and 2 RCTs in which systemic antibiotics supported mechanical debridement. The 2 cohort studies were about mechanical debridement and biofilm control (Table 4).

The selected studies proposed various kinds of treatment, including sodium hypochlorite gel, a desiccant agent, chlorhexidine, triclosan, chitosan brushes, probiotics, diode laser therapy, photodynamic therapy, air polishing, and antibiotics. Most of these consisted of mechanical debridement combined with an additional therapy, such as sodium hypochlorite gel, a desiccant agent, chlorhexidine, probiotics, photodynamic therapy, an enamel matrix derivative, and systemic azithromycin (Table 5).

Table 5. Treatment proposed and related results and conclusions

\begin{tabular}{|c|c|c|c|c|c|c|c|}
\hline Treatment & $\begin{array}{l}\text { Authors, year } \\
\text { of publication }\end{array}$ & Study type & Study description & $\begin{array}{l}\text { Sample } \\
\text { size }\end{array}$ & $\begin{array}{l}\text { Implant } \\
\text { number }\end{array}$ & Results & Conclusions \\
\hline $\begin{array}{l}\text { Sodium } \\
\text { hypochlorite } \\
\text { gel }\end{array}$ & $\begin{array}{l}\text { lorio-Siciliano } \\
\text { et al. } \\
2020^{28}\end{array}$ & $\begin{array}{l}\text { triple-blind } \\
\text { RCT } \\
\text { 6-month } \\
\text { follow-up }\end{array}$ & $\begin{array}{c}\text { mechanical debridement } \\
\text { with sodium hypochlorite } \\
\text { gel } \\
\text { (test group) } \\
\text { vs. } \\
\text { mechanical debridement } \\
\text { with placebo gel } \\
\text { (control group) }\end{array}$ & 46 & 68 & $\begin{array}{l}\text { PPD decreased in both the } \\
\text { test and control groups } \\
\qquad(p=0.0001 \\
\text { and } p=0.0001 \\
\text { respectively) }\end{array}$ & $\begin{array}{c}\text { a complete } \\
\text { resolution } \\
\text { was not achieved } \\
\text { with either therapy }\end{array}$ \\
\hline $\begin{array}{l}\text { Modifying } \\
\text { the implant- } \\
\text { supported } \\
\text { prosthesis }\end{array}$ & $\begin{array}{l}\text { de Tapia et al. } \\
2019^{24}\end{array}$ & $\begin{array}{c}\text { RCT } \\
\text { 6-month } \\
\text { follow-up }\end{array}$ & $\begin{array}{l}\text { modifying } \\
\text { the prosthesis } \\
\text { to allow better } \\
\text { oral hygiene } \\
\text { (test group) } \\
\text { or not } \\
\text { (control group) }\end{array}$ & $\begin{array}{c}45 \\
\text { test } \\
-24 \\
\text { control } \\
-21\end{array}$ & 145 & $\begin{array}{l}\text { changes in } \mathrm{mBl} \text { in the test } \\
\text { and control groups were } \\
1.14 \text { and } 0.50 \text {, respectively } \\
(p=0.010) \text {, in PPD } \\
-0.31 \mathrm{~mm} \text { and } 0.02 \mathrm{~mm} \text {, } \\
\text { respectively }(p=0.040)\end{array}$ & $\begin{array}{l}\text { modifying the } \\
\text { prosthesis improved } \\
\text { clinical outcomes }\end{array}$ \\
\hline $\begin{array}{l}\text { Topical } \\
\text { desiccant agent } \\
\text { in association } \\
\text { with manual } \\
\text { debridement }\end{array}$ & $\begin{array}{l}\text { Lombardo et al. } \\
2019^{29}\end{array}$ & $\mathrm{RCT}$ & $\begin{array}{l}\text { desiccant agent } \\
\text { after debridement } \\
\text { (test group) } \\
\text { vs. } \\
\text { 1\% chlorhexidine } \\
\text { after debridement } \\
\text { (control group) }\end{array}$ & 23 & 52 & $\begin{array}{l}\text { the test group presented } \\
\text { significantly greater } \\
\text { reductions in } \mathrm{BoP}, \mathrm{mBI} \text {, } \\
\text { VPI, and } \mathrm{mPI} \\
\text { than the control group }\end{array}$ & $\begin{array}{l}\text { a complete } \\
\text { resolution of the } \\
\text { inflammatory } \\
\text { conditions } \\
\text { was not achieved } \\
\text { by either group }\end{array}$ \\
\hline $\begin{array}{l}\text { Chlorhexidine- } \\
\text { containing } \\
\text { brush-on gel }\end{array}$ & $\begin{array}{l}\text { Hallström et al. } \\
2017^{42}\end{array}$ & $\begin{array}{c}\text { double-blind } \\
\text { RCT } \\
\text { 12-week } \\
\text { follow-up }\end{array}$ & $\begin{array}{l}\text { chlorhexidine-containing } \\
\text { brush-on gel used as } \\
\text { an adjuvant to } \\
\text { mechanical debridement }\end{array}$ & 37 & 37 & $\begin{array}{l}\text { the test group presented } \\
\text { a reduction in BoP } \\
\text { after } 4 \text { and } 12 \text { weeks } \\
\text { as compared to } \\
\text { the control group } \\
(p<0.05)\end{array}$ & $\begin{array}{l}\text { the findings } \\
\text { indicate moderate } \\
\text { but significant } \\
\text { improvement in } \\
\text { clinical parameters }\end{array}$ \\
\hline $\begin{array}{l}\text { Chlorhexidine } \\
\text { gel }\end{array}$ & $\begin{array}{l}\text { Heitz-Mayfield } \\
\text { et al. } \\
2011^{46}\end{array}$ & $\mathrm{RCT}$ & $\begin{array}{c}\text { non-surgical debridement } \\
\text { with/without } \\
0.5 \% \text { chlorhexidine gel }\end{array}$ & $\begin{array}{l}29 \\
\text { test } \\
-15 \\
\text { control } \\
-14\end{array}$ & 29 & $\begin{array}{c}\text { at } 1 \text { month and from } \\
1 \text { to } 3 \text { months, there were } \\
\text { statistically significant } \\
\text { reductions } \\
\text { in the mean number } \\
\text { of sites with BoP and } \\
\text { the mean PPD values } \\
\text { at implants } \\
\text { in both groups }\end{array}$ & $\begin{array}{c}\text { adjunctive } \\
\text { chlorhexidine gel } \\
\text { did not improve } \\
\text { the results } \\
\text { as compared to } \\
\text { mechanical cleaning } \\
\text { alone }\end{array}$ \\
\hline
\end{tabular}




\begin{tabular}{|c|c|c|c|c|c|c|c|}
\hline Treatment & $\begin{array}{l}\text { Authors, year } \\
\text { of publication }\end{array}$ & Study type & Study description & $\begin{array}{l}\text { Sample } \\
\text { size }\end{array}$ & \begin{tabular}{|l|} 
Implant \\
number
\end{tabular} & Results & Conclusions \\
\hline $\begin{array}{l}0.03 \% \\
\text { chlorhexidine } \\
\text { and } 0.05 \% \\
\text { cetylpyridinium } \\
\text { mouth rinse }\end{array}$ & $\begin{array}{l}\text { Pulcini et al. } \\
2019^{30}\end{array}$ & $\begin{array}{l}\text { double-blind } \\
\text { RCT } \\
\text { 12-month } \\
\text { follow-up }\end{array}$ & $\begin{array}{c}\text { 0.03\% chlorhexidine and } \\
0.05 \% \text { cetylpyridinium } \\
\text { mouth rinse } \\
\text { vs. } \\
\text { placebo } \\
\text { mouth rinse }\end{array}$ & $\begin{array}{c}46 \\
\text { test } \\
-24 \\
\text { control } \\
-22\end{array}$ & 54 & $\begin{array}{l}\text { a reduction in BoP } \\
\text { in the test group } \\
\qquad(p=0.002) \\
\text { and the control group } \\
\qquad(p>0.05)\end{array}$ & $\begin{array}{l}\text { the use of the } \\
\text { test mouth rinse } \\
\text { demonstrated some } \\
\text { adjunctive benefits } \\
\text { in peri-implant } \\
\text { mucositis treatment }\end{array}$ \\
\hline $\begin{array}{l}0.12 \% \\
\text { chlorhexidine } \\
\text { gluconate }\end{array}$ & $\begin{array}{l}\text { Menezes et al. } \\
2016^{39}\end{array}$ & $\begin{array}{c}\text { RCT } \\
\text { 6-month } \\
\text { follow-up }\end{array}$ & $\begin{array}{c}\text { basic periodontal therapy } \\
\text { with } 0.12 \% \text { chlorhexidine } \\
\text { gluconate mouthwash } \\
\text { vs. } \\
\text { basic periodontal therapy } \\
\text { and placebo } \\
\text { mouthwash }\end{array}$ & 37 & $\begin{array}{c}119 \\
\text { test } \\
-61 \\
\text { control } \\
-58\end{array}$ & $\begin{array}{c}\text { significant improvement } \\
\text { in comparison with baseline, } \\
\text { no significant differences } \\
\text { between } \\
\text { the treatment groups }\end{array}$ & $\begin{array}{l}0.12 \% \text { chlorhexidine } \\
\text { was not } \\
\text { more effective } \\
\text { than placebo }\end{array}$ \\
\hline $\begin{array}{l}\text { Triclosan } \\
\text { dentifrice }\end{array}$ & $\begin{array}{l}\text { Ramberg et al. } \\
2009^{26}\end{array}$ & $\begin{array}{l}\text { double-blind } \\
\text { RCT } \\
\text { 6-month } \\
\text { follow-up }\end{array}$ & $\begin{array}{c}\text { dentifrice containing } \\
\text { triclosan } \\
\text { vs. } \\
\text { sodium fluoride dentifrice }\end{array}$ & 60 & N/A & $\begin{array}{l}\text { subjects with peri-implant } \\
\text { mucositis who used a } 0.3 \% \\
\text { triclosan dentifrice exhibited } \\
\text { significantly fewer } \\
\text { clinical signs } \\
\text { of inflammation } \\
\text { than subjects who } \\
\text { used a regular } \\
\text { fluoride dentifrice }\end{array}$ & $\begin{array}{c}\text { the regular use } \\
\text { of triclosan dentifrice } \\
\text { may reduce } \\
\text { the clinical signs } \\
\text { of inflammation }\end{array}$ \\
\hline $\begin{array}{l}\text { Triclosan- } \\
\text { containing } \\
\text { fluoride } \\
\text { toothpaste }\end{array}$ & $\begin{array}{l}\text { Pimentel et al. } \\
2019^{31}\end{array}$ & $\begin{array}{l}\text { RCT } \\
\text { two 3-week } \\
\text { follow-ups }\end{array}$ & $\begin{array}{c}\text { triclosan/ } \\
\text { fluoride toothpaste } \\
\text { vs. } \\
\text { fluoride toothpaste }\end{array}$ & 26 & N/A & $\begin{array}{l}\text { both groups showed } \\
\text { increases in PI } \\
\qquad(p=0.001)\end{array}$ & $\begin{array}{l}\text { triclosan-containing } \\
\text { toothpaste reduced } \\
\text { the RANKL/OPG ratio }\end{array}$ \\
\hline $\begin{array}{l}\text { Triclosan- } \\
\text { containing } \\
\text { toothpaste }\end{array}$ & $\begin{array}{l}\text { Ribeiro et al. } \\
2018^{35}\end{array}$ & $\begin{array}{c}\text { RCT } \\
\text { 6-week } \\
\text { follow-up }\end{array}$ & $\begin{array}{c}\text { triclosan/ } \\
\text { copolymer/ } \\
\text { fluoride toothpaste } \\
\text { vs. } \\
\text { placebo } \\
\text { fluoride toothpaste }\end{array}$ & 22 & 22 & $\begin{array}{l}\text { both groups showed } \\
\text { increases in PI } \\
\text { at implant sites } \\
\text { from the } 3^{\text {rd }} \text { to the } 21^{\text {st }} \text { day, } \\
\text { avoiding } \\
\text { an increase in BoP } \\
\text { throughout the follow-up } \\
\text { was possible only with } \\
\text { triclosan treatment }\end{array}$ & $\begin{array}{l}\text { triclosan-containing } \\
\text { toothpaste controls } \\
\text { the clinical signs } \\
\text { of inflammation }\end{array}$ \\
\hline Chitosan brush & $\begin{array}{l}\text { Wohlfahrt et al. } \\
2019^{25}\end{array}$ & $\begin{array}{c}\text { RCT } \\
\text { 6-month } \\
\text { follow-up }\end{array}$ & $\begin{array}{l}\text { chitosan brush on } \\
\text { an oscillating dental } \\
\text { handpiece } \\
\text { vs. } \\
\text { titanium curette }\end{array}$ & 11 & 24 & $\begin{array}{l}\text { both groups demonstrated } \\
\text { significant reductions in BoP } \\
\text { between baseline } \\
\text { and } 6 \text { months }\end{array}$ & $\begin{array}{l}\text { a chitosan brush } \\
\text { seems to be } \\
\text { a safe and efficient } \\
\text { device for } \\
\text { the debridement } \\
\text { of dental implants }\end{array}$ \\
\hline Probiotics & $\begin{array}{l}\text { Galofré et al. } \\
2018^{20}\end{array}$ & $\begin{array}{l}\text { triple-blind } \\
\text { RCT }\end{array}$ & $\begin{array}{c}\text { oral probiotic } \\
\text { L. reuteri } \\
\text { as an adjuvant to } \\
\text { non-surgical } \\
\text { mechanical therapy }\end{array}$ & $\begin{array}{l}\quad 44 \\
\text { with peri- } \\
\text { implant } \\
\text { mucositis } \\
-22 \\
\text { with peri- } \\
\text { implantitis } \\
-22\end{array}$ & 44 & $\begin{array}{l}\text { a decrease } \\
\text { of } P \text {. gingivalis } \\
\text { bacterial load } \\
\text { at implant sites } \\
\text { with mucositis } \\
\quad(p=0.031)\end{array}$ & $\begin{array}{l}\text { the probiotic } \\
\text { together with } \\
\text { mechanical therapy } \\
\text { produced additional } \\
\text { improvement over } \\
\text { treatment with } \\
\text { mechanical therapy } \\
\text { alone }\end{array}$ \\
\hline Probiotics & $\begin{array}{l}\text { Peña et al. } \\
2019^{32}\end{array}$ & $\begin{array}{l}\text { triple-blind } \\
\quad \text { RCT } \\
\text { 3-month } \\
\text { follow-up }\end{array}$ & $\begin{array}{c}\text { mechanical debridement } \\
\text { with } 0.12 \% \text { chlorhexidine } \\
\text { and L. reuteri } \\
\text { vs. } \\
\text { mechanical debridement } \\
\text { with } 0.12 \% \text { chlorhexidine }\end{array}$ & 50 & 50 & $\begin{array}{l}\text { after the administration } \\
\text { of } 0.12 \% \text { chlorhexidine, } \\
\text { all clinical parameters } \\
\text { improved } \\
\text { in both groups }\end{array}$ & $\begin{array}{l}\text { the administration } \\
\text { of the probiotic } \\
\text { did not seem } \\
\text { to provide } \\
\text { an additional } \\
\text { clinical benefit }\end{array}$ \\
\hline Probiotics & $\begin{array}{l}\text { Hallström et al. } \\
2016^{43}\end{array}$ & $\begin{array}{l}\text { double-blind } \\
\text { RCT } \\
\text { 26-week } \\
\text { follow-up }\end{array}$ & $\begin{array}{l}\text { probiotic supplements } \\
\text { as an adjuvant to } \\
\text { conventional } \\
\text { management } \\
\text { vs. } \\
\text { placebo }\end{array}$ & 49 & N/A & $\begin{array}{l}\text { after } 4 \text { and } 12 \text { weeks, } \\
\text { BoP and PPD significantly } \\
\text { decreased in both groups } \\
\qquad(p<0.05) \text {, } \\
\text { no significant differences } \\
\text { between } \\
\text { the treatment groups }\end{array}$ & $\begin{array}{c}\text { probiotic } \\
\text { supplements } \\
\text { did not provide } \\
\text { additional } \\
\text { improvement } \\
\text { over placebo }\end{array}$ \\
\hline
\end{tabular}




\begin{tabular}{|c|c|c|c|c|c|c|c|}
\hline Treatment & $\begin{array}{l}\text { Authors, year } \\
\text { of publication }\end{array}$ & Study type & Study description & $\begin{array}{l}\text { Sample } \\
\text { size }\end{array}$ & $\begin{array}{l}\text { Implant } \\
\text { number }\end{array}$ & Results & Conclusions \\
\hline Probiotics & $\begin{array}{c}\text { Flichy-Fernàndez } \\
\text { et al. } \\
2015^{44}\end{array}$ & $\begin{array}{c}\text { double-blind } \\
\text { RCT }\end{array}$ & L. reuteri & 34 & 77 & $\begin{array}{c}\text { after treatment with } \\
\text { the probiotic, patients with } \\
\text { mucositis and without peri- } \\
\text { implant disease } \\
\text { showed improvement } \\
\text { in clinical parameters, } \\
\text { with reductions } \\
\text { in cytokine levels }\end{array}$ & $\begin{array}{l}\text { clinical parameters } \\
\text { improved } \\
\text { after treatment } \\
\text { with the probiotic }\end{array}$ \\
\hline $\begin{array}{l}\text { Probiotics with } \\
\text { photodynamic } \\
\text { therapy }\end{array}$ & $\begin{array}{l}\text { Mongardini et al. } \\
2017^{38}\end{array}$ & $\begin{array}{c}\text { RCT } \\
\text { 6-week } \\
\text { follow-up }\end{array}$ & $\begin{array}{l}\text { L. reuteri } \\
\text { with professionally } \\
\text { administered } \\
\text { plaque removal and } \\
\text { photodynamic therapy }\end{array}$ & 20 & 20 & $\begin{array}{c}\text { no significant differences } \\
\text { in clinical outcomes } \\
\text { between } \\
\text { the treatment groups }\end{array}$ & $\begin{array}{l}\text { the adjunctive use } \\
\text { of the probiotic } \\
\text { did not significantly } \\
\text { improve } \\
\text { clinical outcomes }\end{array}$ \\
\hline $\begin{array}{l}\text { Mechanical } \\
\text { curettage with } \\
\text { photodynamic } \\
\text { therapy }\end{array}$ & $\begin{array}{l}\text { Javed et al. } \\
2017^{21}\end{array}$ & $\begin{array}{c}\text { RCT } \\
\text { 12-week } \\
\text { follow-up }\end{array}$ & $\begin{array}{c}\text { mechanical curettage } \\
\text { with/without } \\
\text { adjunctive antimicrobial } \\
\text { photodynamic therapy }\end{array}$ & $\begin{array}{c}54 \\
\text { test } \\
-28 \\
\text { control } \\
-26\end{array}$ & $\mathrm{~N} / \mathrm{A}$ & $\begin{array}{l}\text { PI and PPD were } \\
\text { significantly higher } \\
\text { in the control group } \\
\quad(p<0.001)\end{array}$ & $\begin{array}{l}\text { mechanical } \\
\text { debridement with } \\
\text { photodynamic } \\
\text { therapy is } \\
\text { more effective in the } \\
\text { treatment of peri- } \\
\text { implant mucositis } \\
\text { in comparison with } \\
\text { mechanical } \\
\text { debridement alone }\end{array}$ \\
\hline $\begin{array}{l}\text { Antimicrobial } \\
\text { photodynamic } \\
\text { therapy }\end{array}$ & $\begin{array}{c}\text { Al Rifaiy et al. } \\
2018^{34}\end{array}$ & $\begin{array}{c}\text { RCT } \\
\text { 12-week } \\
\text { follow-up }\end{array}$ & $\begin{array}{l}\text { mechanical debridement } \\
\text { and photodynamic } \\
\text { therapy } \\
\text { (test group) } \\
\text { vs. } \\
\text { mechanical debridement } \\
\text { (control group) }\end{array}$ & 38 & 65 & $\begin{array}{l}\text { reductions in PI } \\
\qquad(p<0.001) \\
\text { and PPD } \\
\quad(p<0.001) \\
\text { in the test group } \\
\text { as compared to } \\
\text { the control group }\end{array}$ & $\begin{array}{c}\text { antimicrobial } \\
\text { photodynamic } \\
\text { therapy } \\
\text { is more effective } \\
\text { in comparison with } \\
\text { manual } \\
\text { debridement alone }\end{array}$ \\
\hline $\begin{array}{l}\text { Air-abrasive } \\
\text { debridement }\end{array}$ & $\begin{array}{l}\text { Lupi et al. } \\
2017^{40}\end{array}$ & $\begin{array}{c}\text { RCT } \\
\text { 6-month } \\
\text { follow-up }\end{array}$ & $\begin{array}{c}\text { maintenance treatment } \\
\text { with glycine powder } \\
\text { air-abrasive debridement } \\
\text { vs. } \\
\text { manual debridement } \\
\text { and chlorhexidine } \\
\text { administration }\end{array}$ & 46 & 88 & $\begin{array}{l}\text { air-abrasive debridement } \\
\text { significantly improved } \\
\text { PI, BoP, PPD, and BS } \\
(p<0.05)\end{array}$ & $\begin{array}{l}\text { treatment with } \\
\text { glycine powder } \\
\text { seems to be } \\
\text { more effective than } \\
\text { traditional treatment } \\
\text { with plastic curettes } \\
\text { and chlorhexidine }\end{array}$ \\
\hline Air polishing & $\begin{array}{c}\text { Riben-Grundstrom } \\
\text { et al. } \\
2015^{41}\end{array}$ & $\mathrm{RCT}$ & $\begin{array}{c}\text { glycine powder } \\
\text { air polishing } \\
\text { vs. } \\
\text { ultrasonic debridement }\end{array}$ & 37 & 37 & $\begin{array}{l}\text { at } 12 \text { months, there were } \\
\text { statistically significant } \\
\text { reductions } \\
\text { in the mean } \mathrm{PI}, \mathrm{BoP} \\
\text { and the number } \\
\text { of periodontal pockets } \\
\geq 4 \mathrm{~mm} \text { within } \\
\text { the treatment groups } \\
\text { in comparison with baseline }\end{array}$ & $\begin{array}{l}\text { non-surgical } \\
\text { treatment with } \\
\text { air polishing } \\
\text { or ultrasonic } \\
\text { debridement } \\
\text { is effective }\end{array}$ \\
\hline $\begin{array}{l}\text { Enamel matrix } \\
\text { derivative }\end{array}$ & $\begin{array}{l}\text { Kashefimehr et al. } \\
\qquad 2017^{37}\end{array}$ & $\begin{array}{c}\text { double-blind } \\
\text { RCT } \\
\text { 3-month } \\
\text { follow-up }\end{array}$ & $\begin{array}{c}\text { mechanical debridement } \\
\text { with } \\
\text { enamel matrix derivative } \\
\text { vs. } \\
\text { mechanical debridement } \\
\text { alone }\end{array}$ & 41 & 41 & $\begin{array}{l}\text { significant improvement } \\
\text { in terms of BoP and PPD } \\
\text { in the test group } \\
\text { as compared to } \\
\text { the control group } \\
(p<0.0001)\end{array}$ & $\begin{array}{c}\text { complete recovery } \\
\text { was not observed } \\
\text { using either } \\
\text { treatment approach }\end{array}$ \\
\hline $\begin{array}{l}\text { Subgingival } \\
\text { ozone and/or } \\
\text { hydrogen } \\
\text { peroxide }\end{array}$ & $\begin{array}{l}\text { McKenna et al. } \\
2013^{45}\end{array}$ & $\begin{array}{c}\text { double-blind } \\
\text { RCT }\end{array}$ & $\begin{array}{c}\text { effect of subgingival } \\
\text { ozone and/or } \\
\text { hydrogen peroxide } \\
\text { on the development } \\
\text { of peri-implant mucositis }\end{array}$ & 20 & 80 & $\begin{array}{l}\text { significant differences } \\
\text { in plaque and modified } \\
\text { gingival and bleeding } \\
\text { indices were observed } \\
\text { between various kinds } \\
\text { of treatments }\end{array}$ & $\begin{array}{l}\text { ozone showed } \\
\text { great potential for } \\
\text { the management } \\
\text { of peri-implant } \\
\text { mucositis }\end{array}$ \\
\hline
\end{tabular}




\begin{tabular}{|c|c|c|c|c|c|c|c|}
\hline Treatment & $\begin{array}{l}\text { Authors, year } \\
\text { of publication }\end{array}$ & Study type & Study description & $\begin{array}{l}\text { Sample } \\
\text { size }\end{array}$ & \begin{tabular}{l|} 
Implant \\
number
\end{tabular} & Results & Conclusions \\
\hline $\begin{array}{l}\text { Systemic } \\
\text { antibiotics }\end{array}$ & $\begin{array}{c}\text { Hallström et al. } \\
2012^{27}\end{array}$ & $\begin{array}{c}\text { RCT } \\
\text { 6-month } \\
\text { follow-up }\end{array}$ & $\begin{array}{c}\text { non-surgical treatment } \\
\text { of peri-implant mucositis } \\
\text { with/without } \\
\text { systemic antibiotics }\end{array}$ & 48 & N/A & $\begin{array}{l}\text { the statistical analysis } \\
\text { failed to demonstrate } \\
\text { differences in PPD } \\
\text { at } 6 \text { months }\end{array}$ & $\begin{array}{l}\text { no short-term } \\
\text { differences } \\
\text { were found between } \\
\text { the } 2 \text { study groups; } \\
\text { the study does not } \\
\text { provide evidence for } \\
\text { the beneficial effect } \\
\text { of systemic antibiotics }\end{array}$ \\
\hline Azithromycin & $\begin{array}{l}\text { Gershenfeld et al. } \\
\qquad 2018^{33}\end{array}$ & $\begin{array}{c}\text { RCT } \\
\text { 6-month } \\
\text { follow-up }\end{array}$ & $\begin{array}{c}\text { mechanical debridement } \\
\text { and systemic azithromycin } \\
\text { vs. } \\
\text { mechanical debridement } \\
\text { and placebo }\end{array}$ & $\begin{array}{l}17 \\
\text { test } \\
-9 \\
\text { control } \\
-8\end{array}$ & 66 & $\begin{array}{l}\text { the treatment patients } \\
\text { showed a consistently } \\
\text { greater reduction } \\
\text { of gingival inflammation } \\
\text { and improvement } \\
\text { in soft tissue healing } \\
\text { than the control patients }\end{array}$ & $\begin{array}{l}\text { the adjunctive use } \\
\text { of azithromycin } \\
\text { can assist in the } \\
\text { control of peri- } \\
\text { implant mucositis }\end{array}$ \\
\hline $\begin{array}{l}\text { Mechanical } \\
\text { debridement }\end{array}$ & $\begin{array}{l}\text { Serino et al. } \\
2018^{47}\end{array}$ & $\begin{array}{l}\text { 7-month } \\
\text { prospective } \\
\text { cohort study }\end{array}$ & $\begin{array}{l}\text { effect of submucosal } \\
\text { mechanical } \\
\text { instrumentation } \\
\text { following supramucosal } \\
\text { plaque removal }\end{array}$ & 44 & 175 & $\begin{array}{l}\text { at } 1 \text { month following } \\
\text { supramucosal plaque } \\
\text { removal, the number } \\
\text { of treated implants with } \\
\text { BoP was reduced with } \\
\text { a concomitant decrease } \\
\text { in the mean PPD value, } \\
\text { following submucosal } \\
\text { instrumentation, } \\
\text { a further reduction } \\
\text { in BoP was recorded with } \\
\text { a concomitant reduction } \\
\text { in the mean PPD value } \\
\text { at the 7-month examination }\end{array}$ & $\begin{array}{c}\text { improvement } \\
\text { in the clinical } \\
\text { condition } \\
\text { appeared to be } \\
\text { in a large extent } \\
\text { due to supramucosal } \\
\text { plaque removal }\end{array}$ \\
\hline Biofilm control & $\begin{array}{l}\text { Gomes et al. } \\
2015^{48}\end{array}$ & $\begin{array}{l}\text { longitudinal } \\
\text { cohort study }\end{array}$ & $\begin{array}{l}\text { comparison of the } \\
\text { gingival and peri-implant } \\
\text { mucosal inflammatory } \\
\text { response to mechanical } \\
\text { biofilm control }\end{array}$ & 22 & N/A & $\begin{array}{l}\text { VPI, mPI and gingival } \\
\text { bleeding indexes reduced } \\
\text { from day } 0 \text { onward }\end{array}$ & $\begin{array}{c}\text { supragingival/ } \\
\text { supramucosal } \\
\text { biofilm control } \\
\text { benefited } \\
\text { both the teeth } \\
\text { and the implants }\end{array}$ \\
\hline $\begin{array}{l}\text { Photodynamic } \\
\text { therapy }\end{array}$ & $\begin{array}{l}\text { Zeza et al. } \\
2018^{49}\end{array}$ & СCT & $\begin{array}{l}\text { professionally } \\
\text { administered } \\
\text { plaque removal and } \\
\text { photodynamic therapy }\end{array}$ & 20 & 20 & $\begin{array}{l}\text { a reduction in the median } \\
\text { number of BoP sites } \\
\text { around implants } \\
\text { from3.5 to } 2.0 \\
(p=0.030)\end{array}$ & $\begin{array}{c}\text { peri-implant } \\
\text { mucositis can be } \\
\text { effectively treated } \\
\text { with photodynamic } \\
\text { therapy }\end{array}$ \\
\hline $\begin{array}{l}\text { Mechanical } \\
\text { debridement } \\
\text { and } \\
\text { photodynamic } \\
\text { therapy }\end{array}$ & $\begin{array}{l}\text { Al Amri et al. } \\
2016^{50}\end{array}$ & CCT & $\begin{array}{l}\text { mechanical debridement } \\
\text { with/without } \\
\text { photodynamic therapy } \\
\text { in the treatment } \\
\text { of peri-implant } \\
\text { inflammation } \\
\text { in T2DM patients }\end{array}$ & $\begin{array}{c}67 \\
\text { test } \\
-34 \\
\text { control } \\
-33\end{array}$ & N/A & $\begin{array}{l}\text { BoP and PPD were } \\
\text { significantly lower } \\
\text { in the test group than } \\
\text { in the control group } \\
\text { at all follow-ups }\end{array}$ & $\begin{array}{c}\text { in patients with } \\
\text { T2DM, mechanical } \\
\text { debridement } \\
\text { with adjunctive } \\
\text { antimicrobial } \\
\text { photodynamic } \\
\text { therapy } \\
\text { is more effective in } \\
\text { the treatment of peri- } \\
\text { implant inflammation } \\
\text { in comparison with } \\
\text { mechanical } \\
\text { debridement alone }\end{array}$ \\
\hline $\begin{array}{l}\text { Mechanical } \\
\text { debridement } \\
\text { paired with } \\
\text { diode laser } \\
\text { application }\end{array}$ & $\begin{array}{l}\text { Lerario et al. } \\
2016^{51}\end{array}$ & CCT & $\begin{array}{l}\text { conventional treatment } \\
\text { with diode laser } \\
\text { application } \\
\text { (test group) } \\
\text { vs. } \\
\text { conventional treatment } \\
\text { alone (control group) }\end{array}$ & 27 & N/A & $\begin{array}{l}\text { a reduction } \\
\text { of pathological sites } \\
\text { from } 89 \% \text { to } 14.35 \% \\
\text { in the test group and } \\
\text { from } 75.69 \% \text { to } 50 \% \\
\text { in the control group }\end{array}$ & $\begin{array}{c}\text { diode laser } \\
\text { seems to be } \\
\text { a valuable tool } \\
\text { for peri-implant } \\
\text { mucositis treatment }\end{array}$ \\
\hline DMT & $\begin{array}{l}\text { Chan et al. } \\
2019^{52}\end{array}$ & $\begin{array}{l}\text { case-control } \\
\text { study }\end{array}$ & $\begin{array}{l}\text { assessing the modifying } \\
\text { effect of DMT on the } \\
\text { induction and resolution } \\
\text { phases of experimental } \\
\text { peri-implant mucositis at } \\
\text { DMT } \geq 3 \mathrm{~mm} \text { (case) and } \\
\text { DMT } \leq 1 \mathrm{~mm} \text { (control) }\end{array}$ & 19 & N/A & $\begin{array}{l}\text { the removal of the } \\
\text { crown and professional } \\
\text { submucosal cleaning } \\
\text { were necessary } \\
\text { to revert to the baseline } \\
\text { gingival index } \\
\text { in the tested implant }\end{array}$ & $\begin{array}{l}\text { a longer mucosal } \\
\text { tunnel results in a } \\
\text { much more difficult } \\
\text { resolution of peri- } \\
\text { implant mucositis }\end{array}$ \\
\hline
\end{tabular}

L. reuteri - Lactobacillus reuteri; T2DM - type 2 diabetes mellitus; DMT - depth of the implant mucosal tunnel; N/A - data not available; PPD - probing pocket depth; $\mathrm{mBI}$ - modified bleeding index; BoP - bleeding on probing; $\mathrm{VPI}$ - visible plaque index; $\mathrm{mPI}$ - modified plaque index; PI - plaque index; RANKL/OPG - receptor activator of nuclear factor kappa B ligand/osteoprotegerin; P. gingivalis - Porphyromonas gingivalis; BS - bleeding score. 


\section{Discussion}

The prevalence data found in this literature review revealed a wide gap in percentage ranges. This could be due to the relevant heterogeneity of the prevalence reported among the 13 cohort studies and 20 crosssectional studies. Other aspects to consider in order to explain this gap are the sample size and the population observed. Some articles addressed a population composed of smokers or subjects affected by diabetes mellitus; both smoking and diabetes mellitus are well-known periodontal risk factors.

Comparing the results of this review regarding the prevalence of peri-implant mucositis to the prevalence of gingivitis provided by the U.S. National Center for Health Statistics (38.70\% PB), a tight overlap can be observed. ${ }^{9,15}$

According to the available data, the average prevalence values for gingivitis and peri-implant mucositis look very similar. This observation is in contrast with the results of a recent study investigating clinical and biological responses in experimental gingivitis and peri-implant mucositis in humans. ${ }^{16}$ Although less biofilm accumulation was observed at the implant sites, the peri-implant mucosa yielded a higher proportion of BoP sites as compared to the gingiva. ${ }^{16}$ This result probably indicates that less visible plaque accumulation is needed for peri-implant mucositis to develop and that the lack of keratinized gingiva, which is a frequent condition around implants, leading to a weaker seal, can contribute to biofilm migration. This would make the onset and progression of peri-implant mucositis easier and faster than in the case of gingivitis. A possible explanation of this discrepancy is that signs of peri-implant mucositis are generally rarely identified because of the great morphological variability of the overhanging prosthesis.

With regard to prosthodontics, it must be emphasized that it definitely plays a crucial role in mucosal homeostasis. Design, structural connections and constituent materials are all factors concretely correlated to plaque accumulation and the soft tissue response. This heterogeneity may help explain the wide gap in peri-implant mucositis percentage ranges found in this review.

During the present investigation, a general deficiency of the available data on this topic emerged, suggesting more focused research is needed in the future, with a general recommendation for more detailed information in the upcoming studies about peri-implant mucositis.

Another relevant aspect concerns the varying clinical indicators used by different studies. Plaque index (PI), BoP, probing pocket depth (PPD), and marginal recession are not always accompanied by radiological examinations to exclude the presence of peri-implantitis. Therefore, it is advisable to collect all the biometric parameters of signs of inflammation, such as redness, swelling, bleeding, and suppuration, and support them with periodontal indices (BoP and PPD) and radiographic examinations. ${ }^{17}$
These limitations are stressed and partially addressed by the 2017 classification of periodontal and peri-implant diseases and conditions. ${ }^{18}$ It is literally cited that "a local dot of bleeding resulting from probing may be the result of a traumatic probing that should not be considered, in the absence of other inflammatory changes, a definitive criterion to characterize a peri-implant soft tissue lesion". For a correct examination, it is consequently crucial to perform circumferential peri-implant probing, using the walking probe method, and to collect all clinical and radiographic parameters to evaluate them as a whole before formulating a diagnosis.

Therefore, considering that attaining a peri-implant mucositis diagnosis seems more complex than a gingivitis diagnosis, the above reported similar prevalence data leads one to presume that the peri-implant mucositis prevalence rates might be underestimated, resulting in a lower clinical perception of this pathology.

Peri-implant mucositis treatment protocols should focus on infection control and the decontamination of the implant surface. Bacterial plaque and calculus must be professionally removed, and the patient must be instructed and motivated to perform proper oral hygiene procedures at home. While gingivitis treatment could achieve restitutio ad integrum through professional hygiene care, mechanical debridement and comprehensive home care, peri-implant mucositis treatment appears more complex, requiring several treatment modalities and devices. Many treatment procedures are performed in association with mechanical debridement, using ultrasonic devices with dedicated polyetheretherketone-coated tips and implantfriendly instruments, such as titanium-coated, carbonfiber, teflon, and plastic curettes. Also, air-abrasive devices or lasers can be used in conjunction with local antibiotics or antiseptics. ${ }^{11,19}$

In the treatment of gingivitis, scaling and periodontal debridement are able to remove bacterial plaque and calculus from the tooth surfaces, allowing proper healing. None of the proposed therapies for peri-implant mucositis presented in this review led to a complete or strongly predictable resolution, but mechanical debridement accompanied by an adjunctive therapy, such as probiotics, chlorhexidine or photodynamic therapy, proved to provide additional improvement over mechanical debridement alone. ${ }^{20,21}$ Galofré et al. compared the effect of the oral probiotic Lactobacillus reuteri as an adjuvant to non-surgical mechanical therapy. ${ }^{20}$ In their triple-blind $\mathrm{RCT}$, oral probiotics and mechanical therapy together produced additional improvement over treatment with mechanical therapy alone. ${ }^{20}$ Also, Javed et al. investigated the outcome of mechanical curettage with or without the adjunct of antimicrobial photodynamic therapy. ${ }^{21}$ Forty-four patients were involved in this RCT study, and after 12 weeks of follow-up, mechanical debridement with photodynamic therapy was determined to be more 
effective in the treatment of peri-implant mucositis as compared with mechanical debridement alone. ${ }^{21}$

Another promising proposed treatment modality is the use of glycine powder air-polishing devices, which were demonstrated to be as effective as mechanical debridement in a study by Schwarz et al. ${ }^{22}$ The same study group, after an electronic and manual search, selected 7 studies which showed that other therapies added to professionally administered plaque removal were quite promising. ${ }^{23}$

A proper prosthetic design that allows good oral hygiene and low plaque accumulation is certainly a key factor in the prevention of peri-implant mucositis. De Tapia et al. reported that when peri-implant tissue inflammation occurs, the prosthetic design should be assessed and modified if necessary to correct the design defects which may be impeding proper hygiene as well as to diminish biomechanical stress factors if involved. ${ }^{24} \mathrm{~A}$ recent $\mathrm{RCT}$ compared peri-implant mucositis treatment through chitosan brushes on oscillating handpieces and titanium curettes; a chitosan brush seems to be a safe and efficient device for the debridement of dental implants. ${ }^{25}$ Likewise, the regular use of a toothpaste containing triclosan appears to be able to reduce the clinical signs of inflammation in the mucosa adjacent to dental implants. ${ }^{26}$ Finally, it has been shown that there is a minimal difference between the non-surgical treatment of peri-implant mucositis with and without systemic antibiotics. ${ }^{27}$

\section{Conclusions}

Currently, the available information on the prevalence rates and the standardized therapeutic protocols for periimplant mucositis are insufficient. Also, it can be presumed that the prevalence rates may be underestimated due to difficulty with making a clinical diagnosis, leading to a lower level of perception among practitioners.

Peri-implant mucositis is a frequently encountered condition. The absence of effective standardized therapeutic procedures that would result in an empirical choice of therapeutic modalities may lead to diminished effectiveness and unsatisfactory treatment outcomes.

It has to be emphasized that implant placement and prosthetic restorations must allow for proper cleaning and plaque control to prevent peri-implant mucositis.

Further research is needed to improve clinicians' skills in the detection of peri-implant mucositis and to determine effective standardized therapies.

\section{ORCID iDs}

Laura Lo Bianco (1) https://orcid.org/0000-0001-9629-2455 Marco Montevecchi (1) https://orcid.org/0000-0001-7312-802X Michele Ostanello (1) https://orcid.org/0000-0002-2781-574X Vittorio Checchi @ https://orcid.org/0000-0002-3053-5562

\section{References}

1. Checchi V, Mazzoni A, Breschi L, Felice P. Histologic observations of two dental implants retrieved after osseointegration. Int J Periodontics Restorative Dent. 2021;41(1):121-125. doi:10.11607/prd.5102

2. Checchi V, Felice P, Zucchelli G, et al. Wide diameter immediate post-extractive implants vs delayed placement of normaldiameter implants in preserved sockets in the molar region: 1-year post-loading outcome of a randomised controlled trial. Eur J Oral Implantol. 2017;10(3):263-278. PMID:28944355.

3. Ephros H, Kim S, DeFalco R. Peri-implantitis: Evaluation and management. Dent Clin North Am. 2020;64(2):305-313. doi:10.1016/j. cden.2019.11.002

4. Checchi V, Gasparro R, Pistilli R, Canullo L, Felice P. Clinical classification of bone augmentation procedure failures in the atrophic anterior maxillae: Esthetic consequences and treatment options. Biomed Res Int. 2019;2019:4386709. doi:10.1155/2019/4386709

5. Berglundh T, Lindhe J, Ericsson I, Liljenberg B, Thomsen P. The soft tissue barrier at implants and teeth. Clin Oral Implants Res. 1991;2(2):81-90. doi:10.1034/j.1600-0501.1991.020206.x

6. Berglundh T, Lindhe J, Jonsson K, Ericsson I. The topography of the vascular systems in the periodontal and peri-implant tissues in the dog. J Clin Periodontol. 1994;21(3):189-193. doi:10.1111/j.1600-051x.1994. tb00302.x

7. Heitz-Mayfield LJA, Salvi GE. Peri-implant mucositis. J Periodontol. 2018;89(Suppl 1):S257-S266. doi:10.1002/JPER.16-0488

8. Schwarz F, Derks J, Monje A, Wang HL. Peri-implantitis. J Periodontol. 2018;89(Suppl 1):S267-S290. doi:10.1002/JPER.16-0350

9. Passariello C, Di Nardo D, Testarelli L. Inflammatory periimplant diseases and the periodontal connection question. Eur J Dent. 2019;13(1):119-123. doi:10.1055/s-0039-1688525

10. Renvert S, Persson GR, Pirih FQ, Camargo PM. Peri-implant health, peri-implant mucositis, and peri-implantitis: Case definitions and diagnostic considerations. J Periodontol. 2018;89(Suppl 1):S304-S312. doi:10.1002/JPER.17-0588

11. Checchi V, Racca F, Bencivenni D, Lo Bianco L. Role of dental implant homecare in mucositis and peri-implantitis prevention: A literature overview. Open Dent J. 2019;13:470-477. doi:10.2174/1874210601913010470

12. Jepsen S, Berglundh T, Genco R, et al. Primary prevention of periimplantitis: Managing peri-implant mucositis. J Clin Periodontol. 2015;42(Suppl 16):S152-S157. doi:10.1111/jcpe.12369

13. Lang NP, Wilson TG, Corbet EF. Biological complications with dental implants: Their prevention, diagnosis and treatment. Clin Oral Implants Res. 2000;11(Suppl 1):146-155. doi:10.1034/ j.1600-0501.2000.011s1146.x

14. Miller SA, Forrest JL. Enhancing your practice through evidencebased decision making: PICO, learning how to ask good questions. J Evid Based Dent Pract. 2001;1(2):136-141. doi:10.1067/med.2001.118720

15. Kelly JE, Sanchez MJ; National Center for Health Statistics (U.S.). Periodontal disease and oral hygiene among children. United States. Vital Health Stat 11. Data from the Health Examination Center. 1972;117:1-28. PMID:4538240.

16. Meyer S, Giannopoulou C, Courvoisier C, Schimmel M, Müller F, Mombelli A. Experimental mucositis and experimental gingivitis in persons aged 70 or over. Clinical and biological responses. Clin Oral Implants Res. 2017;28(8):1005-1012. doi:10.1111/clr.12912

17. Coli P, Sennerby L. Is peri-implant probing causing over-diagnosis and over-treatment of dental implants? J Clin Med. 2019;8(8):1123. doi:10.3390/jcm8081123

18. Berglundh T, Armitage G, Araujo MG, et al. Peri-implant diseases and conditions: Consensus report of workgroup 4 of the 2017 World Workshop on the Classification of Periodontal and Peri-Implant Diseases and Conditions. J Clin Periodontol. 2018;45(Suppl 20):S286-S291. doi:10.1111/jcpe.12957

19. Figuero E, Graziani F, Sanz I, Herrera D, Sanz M. Management of peri-implant mucositis and peri-implantitis. Periodontol 2000. 2014;66(1):255-273. doi:10.1111/prd.12049

20. Galofré M, Palao D, Vicario M, Nart J, Violant D. Clinical and microbiological evaluation of the effect of Lactobacillus reuteri in the treatment of mucositis and peri-implantitis: A triple-blind randomized clinical trial. J Periodontal Res. 2018;53(3):378-390. doi:10.1111/jre.12523 
21. Javed F, BinShabaib MS, Alharthi SS, Qadri T. Role of mechanical curettage with and without adjunct antimicrobial photodynamic therapy in the treatment of peri-implant mucositis in cigarette smokers: A randomized controlled clinical trial. Photodiagnosis Photodyn Ther. 2017;18:331-334. doi:10.1016/j.pdpdt.2017.04.015

22. Schwarz F, Becker K, Renvert S. Efficacy of air polishing for the nonsurgical treatment of peri-implant diseases: A systematic review. J Clin Periodontol. 2015;42(10):951-959. doi:10.1111/jcpe.12454

23. Schwarz F, Becker K, Sager M. Efficacy of professionally administered plaque removal with or without adjunctive measures for the treatment of peri-implant mucositis. A systematic review and meta-analysis. J Clin Periodontol. 2015;42(Suppl 16):S202-S213. doi:10.1111/jcpe.12349

24. de Tapia B, Mozas C, Valles C, Nart J, Sanz M, Herrera D. Adjunctive effect of modifying the implant-supported prosthesis in the treatment of peri-implant mucositis. J Clin Periodontol. 2019;46(10):1050-1060. doi:10.1111/jcpe.13169

25. Wohlfahrt JC, Aass AM, Koldsland OC. Treatment of peri-implant mucositis with a chitosan brush - a pilot randomized clinical trial. Int J Dent Hyg. 2019;17(2):170-176. doi:10.1111/idh.12381

26. Ramberg P, Lindhe J, Botticelli D, Botticelli A. The effect of a triclosan dentifrice on mucositis in subjects with dental implants: A sixmonth clinical study. J Clin Dent. 2009;20(3):103-107. PMID:19711612.

27. Hallström H, Persson GR, Lindgren S, Olofsson M, Renvert S. Systemic antibiotics and debridement of peri-implant mucositis. A randomized clinical trial. J Clin Periodontol. 2012;39(6):574-581. doi:10.1111/j.1600-051X.2012.01884.x

28. Iorio-Siciliano V, Blasi A, Stratul SI, et al. Anti-infective therapy of periimplant mucositis with adjunctive delivery of a sodium hypochlorite gel: A 6-month randomized triple-blind controlled clinical trial. Clin Oral Investig. 2020;24(6):1971-1979. doi:10.1007/s00784-019-03060-2

29. Lombardo G, Signoriello A, Corrocher G, et al. A topical desiccant agent in association with manual debridement in the initial treatment of peri-implant mucositis: A clinical and microbiological pilot study. Antibiotics (Basel). 2019;8(2):82. doi:10.3390/antibiotics8020082

30. Pulcini A, Bollaín J, Sanz-Sánchez I, et al. Clinical effects of the adjunctive use of a $0.03 \%$ chlorhexidine and $0.05 \%$ cetylpyridinium chloride mouth rinse in the management of peri-implant diseases: A randomized clinical trial. J Clin Periodontol. 2019;46(3):342-353. doi:10.1111/jcpe.13088

31. Pimentel SP, Ribeiro FV, Casarin RC, et al. Triclosan-containing fluoride toothpaste on clinical parameters and osteo-inflammatory mediators when applied in a stent during experimental peri-implant mucositis in smokers. Clin Oral Implants Res. 2019;30(2):187-195. doi:10.1111/clr.13405

32. Peña M, Barallat L, Vilarrasa J, Vicario M, Violant D, Nart J. Evaluation of the effect of probiotics in the treatment of peri-implant mucositis: A triple-blind randomized clinical trial. Clin Oral Investig. 2019;23(4):1673-1683. doi:10.1007/s00784-018-2578-8

33. Gershenfeld L, Kalos A, Whittle T, Yeung S. Randomized clinical trial of the effects of azithromycin use in the treatment of peri-implantitis. Aust Dent J. 2018;63(3):374-381. doi:10.1111/adj.12614

34. Al Rifaiy MQ, Qutub OA, Alasqah MN, Al-Sowygh ZH, Mokeem SA, Alrahlah A. Effectiveness of adjunctive antimicrobial photodynamic therapy in reducing peri-implant inflammatory response in individuals vaping electronic cigarettes: A randomized controlled clinical trial. Photodiagnosis Photodyn Ther. 2018;22:132-136. doi:10.1016/j.pdpdt.2018.03.002

35. Ribeiro FV, Casati MZ, Casarin RC, et al. Impact of a triclosancontaining toothpaste during the progression of experimental peri-implant mucositis: Clinical parameters and local pattern of osteo-immunoinflammatory mediators in peri-implant fluid. J Periodontol. 2018;89(2):203-212. doi:10.1002/JPER.17-0302

36. Al Ghazal L, O'Sullivan J, Claffey N, Polyzois I. Comparison of two different techniques used for the maintenance of peri-implant soft tissue health: A pilot randomized clinical trial. Acta Odontol Scand. 2017;75(7):542-549. doi:10.1080/00016357.2017.1352101

37. Kashefimehr A, Pourabbas R, Faramarzi M, et al. Effects of enamel matrix derivative on non-surgical management of peri-implant mucositis: A double-blind randomized clinical trial. Clin Oral Investig. 2017;21(7):2379-2388. doi:10.1007/s00784-016-2033-7
38. Mongardini C, Pilloni A, Farina F, Di Tanna G, Zeza B. Adjunctive efficacy of probiotics in the treatment of experimental peri-implant mucositis with mechanical and photodynamic therapy: A randomized, cross-over clinical trial. J Clin Periodontol. 2017;44(4):410-417. doi:10.1111/jcpe.12689

39. Menezes KM, Fernandes-Costa AN, Silva-Neto RD, Calderon PS, Gurgel BCV. Efficacy of $0.12 \%$ chlorhexidine gluconate for nonsurgical treatment of peri-implant mucositis. J Periodontol. 2016;87(11):1305-1313. doi:10.1902/jop.2016.160144

40. Lupi SM, Granati M, Butera A, Collesano V, Rodriguez Y Baena R. Air-abrasive debridement with glycine powder versus manual debridement and chlorhexidine administration for the maintenance of peri-implant health status: A six-month randomized clinical trial. Int J Dent Hyg. 2017;15(4):287-294. doi:10.1111/idh.12206

41. Riben-Grundstrom C, Norderyd O, André U, Renvert S. Treatment of peri-implant mucositis using a glycine powder air-polishing or ultrasonic device: A randomized clinical trial. J Clin Periodontol. 2015;42(5):462-469. doi:10.1111/jcpe.12395

42. Hallström $H$, Lindgren $S$, Twetman S. Effect of a chlorhexidinecontaining brush-on gel on peri-implant mucositis. Int J Dent Hyg. 2017;15(2):149-153. doi:10.1111/idh.12184

43. Hallström H, Lindgren S, Widén C, Renvert R, Twetman S. Probiotic supplements and debridement of peri-implant mucositis: A randomized controlled trial. Acta Odontol Scand. 2016;74(1):60-66. doi:10.3109/00016357.2015.1040065

44. Flichy-Fernández AJ, Ata-Ali J, Alegre-Domingo T, et al. The effect of orally administered probiotic Lactobacillus reuteri-containing tablets in peri-implant mucositis: A double-blind randomized controlled trial. J Periodontal Res. 2015;50(6):775-785. doi:10.1111/jre.12264

45. McKenna DF, Borzabadi-Farahani A, Lynch E. The effect of subgingival ozone and/or hydrogen peroxide on the development of peri-implant mucositis: A double-blind randomized controlled trial. Int J Oral Maxillofac Implants. 2013;28(6):1483-1489. doi:10.11607/jomi.3168

46. Heitz-Mayfield LJA, Salvi GE, Botticelli D, Mombelli A, Faddy M, Lang NP; Implant Complication Research Group. Anti-infective treatment of peri-implant mucositis: A randomised controlled clinical trial. Clin Oral Implants Res. 2011;22(3):237-241. doi:10.1111/j.16000501.2010.02078.x

47. Serino G, Wada M. Non-surgical mechanical treatment of peri-implant mucositis: The effect of sub-mucosal mechanical instrumentation following supra-mucosal plaque removal. A 7-month prospective single cohort study. Eur J Oral Implantol. 2018;11(4):455-466. PMID:30515485.

48. Gomes SC, Corvello P, Romagna R, Müller LH, Melchiors Angst PD, Oppermann RV. How do peri-implant mucositis and gingivitis respond to supragingival biofilm control - an intra-individual longitudinal cohort study. Eur J Oral Implantol. 2015;8(1):65-73. PMID:25738180.

49. Zeza B, Farina R, Pilloni A, Mongardini C. Clinical outcomes of experimental gingivitis and peri-implant mucositis treatment with professionally administered plaque removal and photodynamic therapy. Int J Dent Hyg. 2018;16(2):e58-e64. doi:10.1111/idh.12302

50. Al Amri MD, Kellesarian SV, Ahmed A, Al-Kheraif AA, Romanos GE, Javed F. Efficacy of periimplant mechanical debridement with and without adjunct antimicrobial photodynamic therapy in patients with type 2 diabetes mellitus. Photodiagnosis Photodyn Ther. 2016;14:166-169. doi:10.1016/j.pdpdt.2016.04.015

51. Lerario F, Roncati M, Gariffo A, et al. Non-surgical periodontal treatment of peri-implant diseases with the adjunctive use of diode laser: Preliminary clinical study. Lasers Med Sci. 2016;31(1):1-6. doi:10.1007/s10103-015-1785-7

52. Chan D, Pelekos G, Ho D, Cortelini P, Tonetti MS. The depth of the implant mucosal tunnel modifies the development and resolution of experimental peri-implant mucositis: A case-control study. J Clin Periodontol. 2019;46(2):248-255. doi:10.1111/jcpe.13066 